IdeAs

Idées d'Amériques

16 | 2020

Les marges créatrices : intellectuel.le.s afrodescendant.e.s et indigènes auX Amériques, XIX-XXe siècle

\title{
Ecos de la Comunalidad en Oaxaca a inicios del siglo $\mathrm{XXI}$
}

Ecos da Comunalidad em Oaxaca no começo do século XXI

Echoes of the Comunalidad in Oaxaca at the beginning of the XXI century

\section{Elena Nava Morales}

\section{OpenEdition}

\section{Journals}

Edición electrónica

URL: http://journals.openedition.org/ideas/9121

DOI: 10.4000/ideas.9121

ISSN: 1950-5701

Editor

Institut des Amériques

Referencia electrónica

Elena Nava Morales, «Ecos de la Comunalidad en Oaxaca a inicios del siglo xx। », IdeAs [En línea], 16 | 2020, Publicado el 01 octubre 2020, consultado el 18 octubre 2020. URL : http:// journals.openedition.org/ideas/9121; DOI : https://doi.org/10.4000/ideas.9121

Este documento fue generado automáticamente el 18 octubre 2020.

\section{) ()ㅛ $\ominus$}

IdeAs - Idées d'Amériques est mis à disposition selon les termes de la licence Creative Commons Attribution - Pas d'Utilisation Commerciale - Pas de Modification 4.0 International. 


\title{
Ecos de la Comunalidad en Oaxaca a inicios del siglo XXI
}

\author{
Ecos da Comunalidad em Oaxaca no começo do século XXI \\ Echoes of the Comunalidad in Oaxaca at the beginning of the XXI century
}

\author{
Elena Nava Morales
}

\section{Introducción}

Este artículo tiene como objetivo central mostrar cómo la categoría de comunalidad hace eco en la actualidad en proyectos de gestión cultural (audiovisual/cinematográfica) y educativos. Elegí el Campamento Audiovisual Itinerante (CAI) y la Universidad Autónoma Comunal de Oaxaca (UACO) como dos ejemplos de estos proyectos dónde la comunalidad se hace presente a manera de eje rector y de filosofía de vida de los pueblos indígenas en Oaxaca. Con esto no pretendo romantizar la comunalidad ni tratarla como una panacea decolonial que alivie todos los males del colonialismo. Mi intención en este texto es hacer un muy breve recorrido del surgimiento de la categoría, reflexionando sobre el contexto que permitió su emergencia en los "márgenes" y cómo esto promovió la sistematización propia y creativa del conocimiento. Teniendo este telón de fondo, problematizaré la continuidad de la comunalidad en nuevas voces ${ }^{1}$ para entender cómo los contextos actuales permiten que esta categoría transite e influya en esferas políticas que están vinculadas al "centro". Esto a su vez nos interpela sobre que tanto los “márgenes" están presentes en el "centro" como viceversa.

2 La metodología seguida para la obtención de los datos presentados en este artículo fue el trabajo de campo no continuo de larga duración ${ }^{2} \mathrm{y}$, específicamente, un trabajo de campo realizado en abril y mayo de 2019; la observación y participación en diversas actividades en algunas comunidades del estado de Oaxaca, entre ellas Guelatao de Juárez y Santa María Tlahuitoltepec Mixe, pueblos que vieron nacer, respectivamente, a Jaime Martínez Luna y a Floriberto Díaz Gómez. También realicé dos entrevistas con actores vinculados a los dos proyectos que menciono en el texto. Además, revisé 
periódicos de circulación nacional y local y una entrevista realizada a Luna Marán en YouTube por el colectivo dedicado a la comunicación: Voces de Oaxaca Periodismo Colectivo donde la entrevistada explica algunos detalles sobre el Campamento Audiovisual Itinerante. El artículo está dividido de la siguiente manera: 1) introducción, 2) el contexto general, 3) la primera parte donde analizo de manera breve el origen y las características de la comunalidad, 4) la segunda parte donde presento los dos proyectos en los que aparece la comunalidad como eje rector $\mathrm{y}$, finalmente, 5) las conclusiones.

\section{Contexto general}

3 A finales de la década de 1970, emergieron procesos de organización comunitaria y una fuerte lucha en defensa de los territorios indígenas en la Sierra Norte del estado mexicano de Oaxaca ${ }^{3}$. Como fruto de estos procesos de defensa de la vida ante intereses gubernamentales locales y nacionales observamos la emergencia de pensadores zapotecos y mixes que sistematizaron el conocimiento local, dando como resultado el surgimiento de la "comunalidad" (Martínez Luna J., 2010; Díaz Gómez F., 2007; Maldonado B., 2011; Aquino A., 2013; Nava E., 2013; Guerrero A., 2013).

Dos antropólogos y pensadores indígenas, Floriberto Díaz Gómez (1951-1995) y Jaime Martínez Luna, (n. 1951) situados en los márgenes de la sociedad mexicana envolvente, acuñaron este término para poder describir y traducir sus realidades, posicionándose frente a los embates del Estado nacional y sus proyectos de desarrollo. Esta categoría, más que ser un concepto que describiera una realidad estática, apuntaba a la importancia de las prácticas y acciones cotidianas del vivir de los habitantes de la Sierra Norte de Oaxaca, tornándola una categoría en movimiento, una categoría vívida y en constante transformación.

5 Desde la década de 1970 hasta la actualidad, la comunalidad ha tomado caminos diversos, se ha reflexionado sobre ella desde diversos ángulos ${ }^{4}$, ha viajado por varias latitudes a través de las voces de personas involucradas en los movimientos indígenas de América Latina o de redes académicas y activistas. Ha impactado las estructuras del pensamiento hegemónico ${ }^{5}$, abonando las discusiones sobre poscolonialidad y decolonialidad, como lo muestra el número 34 de la Revista Cuadernos del Sur ${ }^{6}$, dedicado a la reflexión sobre la comunalidad, en el cual participé.

6 A inicios del siglo xxI, la comunalidad ha tomado fuerza, retomada por nuevas generaciones de intelectuales, jóvenes y activistas vinculados a las luchas pasadas y presentes. También observamos cómo la categoría, de haber surgido en el contexto de las comunidades indígenas de la Sierra Norte, ha ido penetrando y de cierta forma influyendo en algunas dimensiones vinculadas con el centro, como son el ámbito académico o el gubernamental.

7 Me parece que las ideas de Das V. y Poole D. (2008) son apropiadas para poder pensar los conceptos de centro y margen. Específicamente el abordaje antropológico que las autoras proponen permite ver al centro desde la perspectiva de los márgenes. En el libro que ellas compilan propusieron a los autores mirar sus casos desde los márgenes, para poder pensar en cómo las prácticas y políticas de vida de estos espacios moldean o influyen las prácticas políticas de regulación que configuran al Estado (2008: 3). Es decir, cómo desde los márgenes se puede influir en la normatividad dominante encarnada en el Estado. Me parece también que el enfoque antropológico y la propuesta 
de las autoras nos deja mirar que los márgenes no son espacios estáticos, sino lugares donde la acción política, las reconfiguraciones sociales y la sistematización local y creativa de conocimientos permite mover algunas arterias del Estado.

8 Antes de iniciar con la primera parte del artículo es importante explicar brevemente algunos detalles del contexto local. El estado de Oaxaca se ubica al sureste de México y tiene 570 municipios. Se conoce que Oaxaca tiene ocho regiones: Cañada, Costa, Cuenca del Papaloapan, Istmo, Mixteca, Sierra Norte, Sierra Sur y Valles Centrales. El último censo del INEGI de 2015 presenta un total de población estatal de 3. 976.297 habitantes del cual 1.165. 186, mayores de 5 años, hablan una lengua indígena. A lo largo del estado se asientan 16 pueblos indígenas: amuzgo, chatino, chinanteco, chocho, chontal de Oaxaca, cuicateco, huave, ixcateco, mazateco, mixe, mixteco, nahua, triqui, tzotzil, zapoteco y zoque (Barabas A., 2004). En este contexto pluriétnico podemos observar cómo históricamente se han tejido diversos procesos de resistencia, de luchas, de construcciones identitarias, de conflictos interétnicos y de formas de organización propias. La Constitución Política del estado de Oaxaca en su artículo 16, reconoce su composición étnica, el derecho a la libre determinación de los pueblos y comunidades indígenas y su autonomía. También reconoce que los pueblos tienen personalidad jurídica y formas propias de organización social, de gobierno y política. Además, en la Constitución son reconocidos los Sistemas Normativos Internos (antes Usos y Costumbres) de las comunidades, sus territorios y el acceso a sus recursos naturales, así como sus formas religiosas, educativas y artísticas. Esto nos coloca frente a un estado que históricamente ha ido a la vanguardia de las legislaciones sobre los derechos colectivos de los pueblos indígenas (Mesri Hashemi-Dilmaghani P., 2013).

\section{Primera parte}

\section{Los caminos de la comunalidad}

9 A finales de enero del 2016, entrevisté a Jaime Martínez Luna quién me contó cómo nació la comunalidad. Decía que entre 1977 y 1981 el término apareció desde la dimensión de la oralidad. Es decir, no fue un término que naciera escrito, sino hablado. Se fortaleció cuando Jaime Martínez Luna se encontró con Floriberto Díaz Gómez, ambos como guías de sus organizaciones ${ }^{7}$, la Organización en Defensa de los Recursos Naturales y Desarrollo Social de la Sierra Juárez (Odrenasij) y el Comité Coordinador para la Defensa de los Recursos Naturales, Humanos y Culturales de la Región Mixe (Codremi), respectivamente 8 . Cada uno ponía énfasis en diferentes dimensiones de la categoría. Por ejemplo, Floriberto estaba más interesado en la cuestión del territorio y de la educación, mientras Jaime se preocupó más por la fiesta, el tequio ${ }^{9}$ y la comunicación.

Desde un marco más amplio y sin perder de vista la dimensión histórica, es importante resaltar que la comunalidad tiene como cuna al movimiento campesino indígena de la Sierra Norte de Oaxaca, en el cual se observa un complejo panorama de organización etnopolítica. También es central mencionar que la región de la Sierra Norte tiene recursos forestales y mineros y el $99 \%$ de la tierra es comunal. Esto explica por qué las luchas de los pueblos se inspiraron principalmente en demandas territoriales, pero también de abastecimiento de la región, educación, salud, autonomía con respecto a las cabeceras distritales, etc. Aquino A. lo explica con precisión: 
Estas luchas forman parte de los esfuerzos de los pueblos de toda América Latina en contra del colonialismo interno, es decir, se trata de luchas que desde lo local han intentado transformar las relaciones de dominación entre el Estado y los pueblos originarios. (2013: 8)

11 De esta manera, Floriberto Díaz y Jaime Martínez reflexionaron sobre la comunalidad como una referencia de sus luchas y de sus maneras de vivir en comunidad. Primero, permitiéndose analizar su propia realidad desde sus propias voces; segundo, construyendo categorías desde los ámbitos locales para describir sus prácticas políticoculturales como la asamblea, el tequio o la fiesta; tercero, posicionando la categoría como bandera de un movimiento etnopolítico. Los movimientos etnopolíticos podrían ser entendidos como "las afirmaciones protagónicas de la etnicidad, estructuradas en forma de organizaciones no tradicionales orientadas hacia la defensa de los intereses de los grupos étnicos." (Bartolomé M., 1997: 166).

Para el caso de Floriberto Díaz Gómez la comunalidad tiene como base el trabajo que desarrolló en su pueblo (Santa María Tlahuitoltepec Mixe) y en su región, después de regresar de la ciudad de México donde estudió antropología en la Escuela Nacional de Antropología e Historia en los años setenta y también después de haber sido seminarista. Ambas influencias salen a relucir de manera clara en la configuración de su comunalidad. La comunalidad para Floriberto:

Expresa principios y verdades universales en lo que respecta a la sociedad indígena, la que habrá que entenderse de entrada no como algo opuesto, sino como diferente de la sociedad occidental. Para entender cada uno de sus elementos hay que tener en cuenta ciertas nociones: lo comunal, lo colectivo, la complementariedad y la integridad. Sin tener presente el sentido comunal e integral de cada parte que pretendamos comprender y explicar, nuestro conocimiento estará siempre limitado.

(Díaz F., 2007:40)

13 Así mismo, la comunalidad para Floriberto Díaz tiene cinco elementos fundamentales presentes en las comunidades mesoamericanas: la tierra, el consenso en asamblea para tomar decisiones, el ejercicio de la autoridad como un servicio gratuito, el trabajo colectivo (tequio) y los ritos y ceremonias. Una de las principales contribuciones de Floriberto radica en que a través del conocimiento que sistematizó, podemos asomarnos de cerca a la episteme ayuujk desde la visión de un insider.

14 Jaime Martínez Luna nació en Guelatao de Juárez, al igual que Floriberto Díaz, pisó los terrenos de la antropología. Se formó como antropólogo en la Universidad Veracruzana, en el estado de Veracruz. Para Jaime Martínez Luna:

La comunalidad, que es nuestra manera de pensar, se origina en la historia del despojo; en la obligada relación que hemos mantenido con los territorios que nos dejó la conquista y la explotación voraz de la tierra. Es decir, la comunalidad es también fruto de la resistencia a la historia colonial. (Martínez Luna J., 2010: 45)

En la comunalidad de Jaime encontramos diversos elementos como son la naturolatría, la comunalicracia, el derecho y la cultura propios, la tecnología, la resistencia, la unidad de todos, la adecuación, la compartencia, entre otras categorías que configuran su andamiaje conceptual (Martínez Luna J., 2010 y 2003).

Uno de los aportes más interesantes de Martínez Luna es transformar la comunalidad en verbo, es decir, pensar en comunalizar. Comunalizar se refiere a poder practicar la comunalidad dentro de las estructuras políticas, organizativas o de la vida diaria de los pueblos. Comunalizar sería una especie de indigenización (Salhins M., 1999) a partir de 
los preceptos de la comunalidad ${ }^{10}$. Comunalizar abre caminos para reflexionar críticamente sobre las maneras occidentales de concebir el mundo de manera homogénea y cuestionar valores e ideologías como el individualismo o la propiedad privada, entre otros.

Al hablar de la comunalidad de Jaime y de la comunalidad de Floriberto no estoy queriendo separarlas radicalmente, simplemente intento diferenciarlas pues aunque los dos son serranos hay diferencias que marcan sutilezas importantes. El concepto de Haraway D. (1995) de conocimiento situado es importante aquí para explicar cómo los conocimientos están estrechamente vinculados a la subjetividad y entorno de sus emisores pues sus contextos abrazan ciertas particularidades. Las historias de vida de cada uno de estos pensadores marcan de manera determinante la forma en que construyeron el concepto de comunalidad. Cada uno tiene un origen étnico distinto, Floriberto Díaz es ayuujk, Jaime Martínez Luna es zapoteco, las maneras en que conciben el territorio, las fiestas, el tequio, la espiritualidad o el sistema de cargos están vinculadas a ethos diferentes.

Es importante decir también que la comunalidad no es un pensamiento cuyos orígenes sean esencialmente indígenas, se constituye del conjunto de aprendizajes y vivencias que los dos pensadores tuvieron en el camino. Por ejemplo, en el caso de Jaime Martínez Luna hay una fuerte influencia de los autores y pensadores que leyó durante su paso por la Universidad Veracruzana:

Martínez Luna reconoce las influencias e inspiraciones en sus escritos. De la historia sobresalen académicos mexicanos como Miguel León Portilla, Enrique Florescano, Teresa Rojas, Alfredo López Austin, María de los Ángeles Romero, entre otros. De la antropología mexicana clásica reconoce a Guillermo Bonfil Batalla (del cual ciertamente retoma la noción de lo propio) y a Arturo Warman. Otros autores como Raimon Panikkar y Edgar Morin también aparecen en su repertorio. Entre los autores oaxaqueños que fueron manantial de sus reflexiones tenemos a Floriberto Díaz (ayuujk), Joel Aquino (zapoteco), Marcos Sandoval (triqui) y Benjamín Maldonado. La influencia marxista también deja sus huellas en la obra de Martínez Luna, entre los autores más importantes están: Carlos Marx, Antonio Gramsci, Louis Althusser, Herbert Marcuse y Lucien Sebag. Pero, al igual que Floriberto Díaz, Jaime Martínez Luna reconoce que la principal influencia en su pensamiento es la gente de su comunidad...

(Nava E., 2018: 34-35)

En esta cita podemos observar que Jaime Martínez Luna tuvo contacto con una importante literatura marxista, pero también influyeron en él pensadores, intelectuales y antropólogos indígenas y no indígenas. Además de lo anterior, el pensamiento de la gente de su pueblo, también influyó en la configuración de su comunalidad. Cuando hablo del pensamiento de su pueblo me refiero a las maneras en que su comunidad, regida por Sistemas Normativos Internos, se organiza cultural y políticamente. Un ejemplo de estas maneras de organizarse es la asamblea comunitaria, órgano máximo de decisión en la comunidad; otro ejemplo es el sistema de cargos, que a través de un sistema escalafonario, hace participar a los comuneros en la administración (civil, religiosa y territorial) del municipio ${ }^{11}$.

Esto no significa que la categoría de comunalidad haya sido transgredida por el "pensamiento" occidental, quiere decir que fue construida por un pensador que, desde los márgenes logró tejer y entrelazar de manera creativa sus conocimientos y aprendizajes tanto dentro como fuera de Guelatao para poder construir una categoría 
que diera cuenta de la realidad vivida por los pueblos indígenas zapotecos de la Sierra Norte de Oaxaca en el último tercio del siglo xx.

21 No es de sorprenderse que desde la década de 1970 en que la comunalidad emanó del pensamiento de Floriberto Díaz y Jaime Martínez Luna, no hubo un reconocimiento por parte de la academia hegemónica de aquella época, Maldonado B. (2003) lo explica de la siguiente manera:

Con el paso de los años, muchos indios y no indios han seguido esta idea de la comunalidad en su trabajo docente, académico o activista, dándole diversas formas; pero no es una teoría que haya tenido buena acogida en el mundo académico mexicano. Originalmente es una propuesta que, en el caso de Oaxaca, fue formulada en la sierra Norte o sierra Juárez hace dos décadas por antropólogos indios.

(Maldonado B. en Martínez Luna J., 2003: 10)

Lo anterior nos remite a los campos de poder donde circulan las disciplinas en los ámbitos universitarios y académicos. Para este caso, es central destacar que siempre ha habido una relación de la antropología mexicana con el Estado mexicano, algunas veces más tensa y algunas más laxa. Lomnitz C. (1996) explica cómo la antropología mexicana ha pasado por diferentes ciclos de descubrimiento/desilusión los cuales deben ser comprendidos respecto a la aparición de normatividades dominantes diferenciadas. Según el autor, en el segundo ciclo de descubrimiento/desilusión de la historia de la antropología mexicana, que inicia cuando la religión pasa a segundo término, el Estado aparece como la nueva normatividad dominante y la antropología funge como una mediadora entre el Estado y el "pueblo". En un tercer ciclo, Lomnitz C. (1996) nos presenta una mediación de la antropología con foco en el mercado del consumo y una disminución del papel del Estado. De esta manera, pensando en el Estado mexicano como una entidad normativa aún dominante, como lo expresa Lomnitz C. (1996), que se reproduce en diversas dimensiones, no podían faltarnos los sistemas académicos nacionales, podríamos decir que la antropología de la época también fue influenciada por los ejes rectores del Estado. Si miramos desde la comunalidad (desde los márgenes) a la antropología mexicana de aquel tiempo observamos sus contradicciones y sus búsquedas. Es decir, mirar desde los márgenes nos ofrece una mirada única para comprender al Estado "not because it captures exotic practices, but because it suggests that such margins are a necessary entailment of the state, much as the exception is a necessary component of the rule" (Das V. y Poole D., 2004: 4)

La academia hegemónica en México no permitió que la comunalidad emergiera como una teoría, como cualquier otra venida del Norte, simplemente la invisibilizó. Y más aún si venía desde los márgenes, de un espacio fuera de la academia, como bien lo explica Martínez Luna:

Había que construir un nuevo lenguaje, la misma práctica de trabajo cotidiano con nuestras comunidades permitió elaborar un concepto natural que no pretendía ningún rigor académico, no pretendía implicar ningún razonamiento positivista.

(Entrevista a Jaime Martínez Luna por La Coperacha, 16 de mayo de 2016)

Con la comunalidad ha sucedido como con los conocimientos de diversas poblaciones que han sido sometidas a las fuerzas coloniales de Occidente, dónde ha prevalecido el epistemicidio. Como bien lo explican los Comaroff:

These other worlds, in short, are treated less as sources of refined knowledge than as reservoirs of raw fact: of the historical, natural, and ethnographic minutiae from which Euromodernity might fashion its testable theories and transcendent truths, its axioms and certitudes, its premises, postulates, and principles.

(Comaroff J. \& Comaroff J., 2012: 1) 
dejar de pensar en el epistemicidio, podemos también observar cómo la comunalidad como un pensamiento venido de los márgenes provoca cierta inquietud y malestar entre algunos representantes de la hegemonía académica/estatal pues genera nuevas maneras de mirar y desestructura formas antiguas de proceder y crear discursos. En cierta medida la comunalidad "domestica" y/o "indigeniza" estas formas occidentales de proceder, de gobernar y de pensar. Albert B. (2000) muestra diversas maneras de indigenización de la modernidad:

Desde os fenônemos de reinvenção cultural (de auto-objetivação ou de "reculturação") até os mecanismos conexos de apropriação sócio-simbólica indígena dos recursos e discursos do mercado, das instituições de desenvolvimento (governamentais ou não) e das tecnologias de comunicação incluíndo-se aí o movimento generalizado de reapropriação dos conceitos antropológicos e dos escritos etnográficos (Thomas N., 1997, p. 373-5).

(Albert B., 2000: 18)

Para finalizar este apartado me parece importante decir que al hablar de "márgenes" y "centro" no estamos intentando reproducir un binarismo, sino usar estas categorías para el análisis y mirar sus posibles traslapes y encuentros.

\section{Segunda parte}

En este segundo momento del texto reflexionaremos sobre el resurgimiento de la comunalidad en nuevas voces. En el primer caso, referente a la Universidad Autónoma Comunal de Oaxaca, observaremos los nuevos contextos que permiten un tránsito de la comunalidad a esferas políticas que están ancladas en el "centro" y cómo la comunalidad sostiene sus principios al encontrarse con los preceptos del "centro", es decir, si pensamos en términos de márgenes, cómo los márgenes interpelan y trastocan el centro. En el segundo ejemplo veremos el viaje de la comunalidad a la dimensión audiovisual a través del proyecto Campamento Audiovisual Itinerante (CAI), el cual se conserva en los márgenes (pero con vínculos con el centro) y busca la reproducción de la categoría, no sólo en las prácticas audiovisuales, sino también en la vida cotidiana de los jóvenes que son seleccionados para participar en el campamento. Retomando a Das V. y Poole D. (2008), debemos considerar que el Estado, en relación con sus márgenes, está permanentemente redefiniendo sus modos de legislar y gobernar. Cabe señalar que los márgenes, para las autoras, también son "sitios de práctica en los que la ley y otras prácticas estatales son colonizadas mediante otras formas de regulación que emanan de las necesidades apremiantes de las poblaciones, con el fin de asegurar la supervivencia política y económica" (Das V. y Poole D., 2008: 24), también agregaría la supervivencia cultural, como veremos en los dos casos presentados en este trabajo.

Los dos ejemplos nos interpelan sobre los "márgenes" y los "centros" y son importantes en la actualidad pues revelarán las maneras en que la comunalidad se ha reproducido y ha transitado por diversas dimensiones.

\section{Universidad Autónoma Comunal de Oaxaca}

El primer ejemplo se inscribe en el terreno de la educación. Durante un trabajo de campo que hice en Guelatao de Juárez en abril de 2019, Jaime Martínez Luna me invitó a una de las reuniones en las que se discutió la propuesta de la Universidad Autónoma 
Comunal de Oaxaca. La reunión se llevó a cabo en el Instituto de Investigaciones Sociológicas de la Universidad Autónoma Benito Juárez de Oaxaca. Había más de 30 personas que participarían en la reunión. Eran algunos representantes de entidades educativas de diferentes pueblos del estado de Oaxaca, también había personas de entidades gubernamentales y de la sociedad civil de Oaxaca y de otros lugares del país. La idea principal era discutir el plan que se iba a seguir para construir un espacio educativo diferente, donde algunos sujetos de los pueblos indígenas en Oaxaca pudieran crear y ordenar sus propios conocimientos. Arturo Guerrero, uno de los principales impulsores de los procesos educativos y de comunicación desde una visión comunitaria en el estado de Oaxaca y que ha trabajado por más de dos décadas con Jaime Martínez Luna, me compartió algunas ideas al respecto:

La comunalidad es el fundamento de esta Universidad y en ese sentido su propia negación, la negación de la universidad como ha sido hasta ahora. La comunalidad es el fundamento que pretende negar la manera de ser de la universidad convencional para aprovechar este espacio y ordenar el conocimiento propio. Apropiarse de una institución como han hecho por siglos las gentes de las comunidades. Lo estamos planteando como uno de los ejes de un proceso formativo mucho más amplio en todo el estado de Oaxaca. La realidad de Oaxaca es comunitaria, entonces partimos de que la gente tiene el conocimiento, pero está roto, son 500 años de negación. Pero el conocimiento sigue ahí y necesita ordenarse y fortalecerse con elementos de otras tradiciones y de otras culturas. La universidad puede ser una vía útil para eso, para hacer lo que los pueblos vienen haciendo desde hace tiempo que es reordenar su conocimiento.

(Entrevista Arturo Guerrero, 19 de junio de 2020).

En la reunión se comentó que, aprovechando la visita del presidente actual de México, Andrés Manuel López Obrador ${ }^{12}$, al pueblo de Guelatao de Juárez, el 21 de marzo de $2019^{13}$, algunos involucrados en este proyecto de la Universidad de la Comunalidad o Universidad Nacional Comunal, como la llamaron inicialmente, entregaron al presidente una primera propuesta para crear la universidad.

31 La idea de la reunión era discutir las maneras en que se iba a constituir una propuesta más formal y aterrizada centrada sobre la universidad, para poder entregarla nuevamente al presidente después de septiembre de 2019. Durante las discusiones en la reunión, una de las ideas que causó revuelo fue que la universidad se estaba construyendo en los márgenes. No porque la idea de margen causara aversión, sino porque el margen apareció como un espacio legítimo desde el cual se puede construir una educación comunitaria. Para ilustrar esta cuestión puedo citar el testimonio de uno de los participantes, representante de la Universidad Comunal Intercultural de Huitepec, del pueblo de San Antonio Huitepec. Este profesor manifestó que en su pueblo necesitaban autonomía: "quiero que me dejen operar mi educación, la clave está en la comunidad, estamos al margen de lo otro".

La idea de margen, durante la reunión, quedó vinculada al proyecto de una universidad comunal (ni privada, ni pública), la cual buscará tener un modelo reticular configurado por redes de espacios de enseñanza-aprendizaje. Según Arturo Guerrero, son quince los espacios que formarán la retícula, se ubican en: Capulálpam de Méndez, San Andrés Solaga, Santa María Yaviche, Santa María Tlahuitoltepec, Comitancillo, Ciudad Ixtepec, San Francisco Ixhuatán, Unión Hidalgo, Matías Romero, San Pedro Amuzgos, San Antonio Huitepec, Tlaxiaco, Jaltepec de Candayoc, Santa María Colotepec y Guelatao de Juárez. 
33 No volví a ser parte de estas reuniones pues tuve que regresar a la capital del país a continuar con mis obligaciones, sin embargo, di seguimiento a la iniciativa por medio de las noticias publicadas en diferentes medios electrónicos de circulación nacional (periódicos y revistas).

34 En agosto de 2019, la diputada local del Distrito XXV Juana Aguilar Espinoza del partido de Morena, presidenta de la Comisión de Educación, Ciencia e Innovación, presentó ante la legislatura del estado de Oaxaca la iniciativa para crear la Universidad Autónoma Comunal como un organismo descentralizado del Estado, desescolarizada, regional y comunitaria. Se propuso que tendría el tequio como base de su funcionamiento, ademas:

Su actividad se basará en la práctica que se desarrolla en la vida comunitaria, en donde la mayor parte de tiempo el estudiante se dedicará a la realización de proyectos concretos de trabajo, lo cual permitirá la no dependencia de instalaciones o infraestructura específica para su realización.

(Matías P., Proceso, 2019)

Algunos de los objetivos que se plantearon eran poder ofrecer una educación que no descansara en las maneras occidentales de sistematizar el conocimiento, sino que potencializara, legitimara y fortaleciera el razonamiento propio de los pueblos indígenas de Oaxaca, Flores R. (2019) en su nota periodística lo explica de la siguiente manera:

De aprobarse sería la primera institución en el país no vinculada al razonamiento eurocéntrico de la educación y la primera experiencia en América Latina que reconozca los conocimientos que se generan en el estado a través de las distintas formas de trabajo y de organización, es decir los pensamientos propios de los pueblos del estado.

(Flores R., Oaxaca Media, 2019)

El día 15 de enero de 2020, con 31 votos a favor, la Legislatura de Oaxaca aprobó la creación de la Universidad Autónoma Comunal de Oaxaca:

Una vez que el decreto entre en vigor, la Comisión Permanente de Educación, Ciencia, Tecnología e Innovación del Congreso local emitirá la convocatoria para la elección del consejo fundador, cuyos cargos serán honoríficos, y su única función será la elección y nombramiento de rector y miembros del consejo académico.

(Matías P., Proceso, 2020).

La Universidad Autónoma Comunal de Oaxaca, reconocida en 2020 constitucionalmente, tendrá una estructura reticular que contemple las experiencias educativas que tienen más de 10 años en diferentes localidades de Oaxaca. Además, su filosofía será trabajar con un modelo que emane de las propias comunidades, de su pensar, de su sentir, de sus cosmovisiones (Flores R., Oaxaca Media, 2020). La Universidad Autónoma Comunal de Oaxaca sería un espacio descentrado, que hará educación desde la comunidad y para la comunidad y fortalecerá los procesos de sistematización de conocimientos locales, como ya han venido haciendo algunas universidades como la Universidad Comunal Intercultural del Cempoaltépetl, en Santa María Tlahuitoltepec Mixe, o la Universidad Comunal Intercultural de Huitepec, en San Antonio Huitepec. La apuesta clara de este proyecto es seguir los principios emanados de la comunalidad. En este sentido observamos cómo los "márgenes" interpelan y trastocan al "centro", es decir desde las acciones de los márgenes hay ciertos movimientos en las entidades, prácticas y discursos que configuran al Estado. 
Para finalizar, es importante dejar claro que la UACO es parte de un proceso largo de negociaciones y tensiones entre Estado y márgenes. Recordemos que a la UACO la antecede la experiencia de la creación de la Licenciatura en Comunalidad y la Maestría en Educación Comunal, fruto de aproximadamente cinco años de trabajo entre la Universidad Autónoma Benito Juárez de Oaxaca (UABJO) y algunos integrantes de la Academia de la Comunalidad ${ }^{14}$. La UACO también ha sido impulsada desde la Academia de la Comunalidad. En palabras de uno de sus miembros:

La relación entre la UACO y la comunalidad es obvia. Es fortalecer el saber y la construcción del conocimiento de los pueblos desde una situación no colonial, pensar cómo le damos la vuelta a la modernidad. Ya tenemos muchos conocimientos pero estamos totalmente perdidos, necesitamos reconocerlos para usarlos de manera adecuada. La idea es dar una formación que no te haga migrar, sino que te arraigue y que se centre en lo importante. Lo importante es dejar la abstracción y volver a lo concreto. Se busca que los contenidos de las licenciaturas tengan una orientación comunitaria pero al mismo tiempo se busca que la universidad misma y lo que ella promueva también tenga el sentido comunal. La idea es inspirarse o poder recrear la organización comunal en la universidad. Crear un espacio donde estos saberes sean legítimos y poder moverte en ese espacio de legitimidad desde tus propios conceptos.

(Entrevista Arturo Guerrero, 19 de junio de 2020).

\section{Campamento Audiovisual Itinerante}

El segundo ejemplo nos muestra cómo generaciones más jóvenes han reproducido la noción de comunalidad y han logrado crear proyectos de gran magnitud, como es el Campamento Audiovisual Itinerante (CAI), un proyecto impulsado hasta el año pasado por la asociación civil "La Calenda Audiovisual" cuyos orígenes datan de 2011 y que también impulsa otros proyectos como la red de cines comunitarios "Aquí Cine".

El Campamento Audiovisual Itinerante es un espacio que sucede una vez al año, en el verano, en comunidades del estado de Oaxaca. Fue fundado por tres productoras y directoras de cine: Luna Marán, Mariana Musalem y Laura Ramírez. En cada edición se convoca a jóvenes de todo el país que están interesados en el arte audiovisual, se conforman grupos heterogéneos de jóvenes de zonas rurales, urbanas, del norte y del sur. Durante tres semanas toman talleres y clases con maestros mexicanos y extranjeros y hacen sus propias producciones, las cuales son difundidas de diferentes maneras, como lo explica Damián López, uno de sus profesores:

Se proyectan al terminar el CAI en una gira que se llama Noches CAI, son proyecciones en varias partes, entre ellas los lugares de donde vienen los participantes. Las producciones se difunden también por Vimeo, Facebook y en las próximas ediciones también se usará YouTube para la difusión.

(Entrevista con Damián López, 2 de marzo de 2020).

41 Algunas de las comunidades de la Sierra Norte donde el CAI ya se llevó a cabo son: Guelatao de Juárez, Capulalpam de Méndez, San Juan Evangelista Analco, San Miguel Amatlán. El primer campamento se realizó en Guelatao de Juárez, pues de ahí es una de sus co-fundadoras: Luna Marán. Se realiza siempre en comunidades de la Sierra Norte porque es una región de gran riqueza cultural y natural, además porque la mayoría de los municipios de la zona se rigen por Sistemas Normativos Internos (antes Usos y Costumbres), lo que promueve cierta seguridad y facilita la comunicación con las autoridades municipales y la organización del campamento. En 2019, el CAI llegó a su octava edición y se realizó en el pueblo de Santa María Albarradas. Generalmente el CAI 
tiene grupos de trabajo fijos como animación, ficción y diseño sonoro, aunque en algunas ocasiones ha tenido otros grupos que no duran las tres semanas pero que también tienen personas inscritas, como actuación o crítica cinematográfica. En los grupos de trabajo siempre hay personas de la misma comunidad interesadas en aprender sobre cine y sobre realización audiovisual. También en el CAI se forma un grupo de niños de la misma comunidad que participan de talleres y acompañan varias actividades, esto los hace involucrase desde pequeños en la realización audiovisual.

El eje rector del campamento es la comunalidad, según el relato del profesor Damián López:

El tronco común del CAI es la comunalidad, la primera parte siempre es de charlas sobre comunalidad, hay una plática con Jaime Martínez Luna, también hay una charla con Yásnaya Aguilar sobre el imaginario del indígena en el cine.

(Entrevista con Damián López, 2 de marzo de 2020).

En otro testimonio, Luna Marán también relata cómo la comunalidad es uno de los ejes rectores y filosóficos del campamento:

La comunalidad es la filosofía base del proyecto. Todos los jóvenes aprenden de la forma de organización de las comunidades, aprenden de su sistema de cargos, del tequio, de la asamblea, de la fiesta como mecanismos que se tienen de cohesión social, de trabajo por el bien común, de realización de acuerdos y todo eso sirve para poder llegar a la producción audiovisual desde otro lado, tratando de eliminar todos los mecanismos de violencia laboral que tiene la industria cinematográfica y toda la cuestión de corrupción que también está implícita.

(Matías P., Portal Página 3, 2018).

La metodología del CAI es el mayor reto que enfrentan año tras año, reunir a jóvenes tan diversos, con distintos orígenes y con personalidades que, en ocasiones, son completamente opuestas. Durante las tres semanas, los participantes deben organizarse en grupos para poder lograr crear un producto que generalmente es una ficción y "deben desprenderse del ego y trabajar desde la colectividad, en ese sentido la comunalidad como una filosofía del colectivo, los ayuda a trabajar" (Entrevista con Damián López, 2 de marzo de 2020).

Siendo la comunalidad uno de los ejes rectores del CAI, los participantes viven en la comunidad durante las tres semanas que dura el campamento, conviven y aprenden de la gente que los recibe y se llevan una diversidad de experiencias sobre las formas de organización de estas comunidades. Es decir, aunque de manera breve, las y los jóvenes experimentan y viven los claroscuros de la comunalidad.

Otra cuestión que es de resaltarse y que coincide con el ejemplo anterior de la Universidad Autónoma Comunal de Oaxaca es que en el CAI los conocimientos propios y las formas locales de ver el mundo son centrales para poder realizar sus proyectos audiovisuales. Lo explica Luna Marán de la siguiente manera:

Lo que nosotros en principio queremos con el campamento es que los jóvenes que quieren producir audiovisual lo puedan hacer desde una visión honesta a su realidad y su contexto, eso implica que sean conscientes de la sabiduría, del talento que tienen por quienes son, por de dónde vengan, por la comunidad a la que pertenezcan, por la cultura en la cual se hayan desarrollado, que aprendan a desarrollar sus historias, historias que son muy ricas porque son muy diversas, porque son únicas. En ese sentido, deseamos que el proyecto a largo o mediano plazo pueda desarrollar producción audiovisual que refleje la diversidad de nuestro país y con esto valorar nuestras culturas.

(Voces de Oaxaca, 3 abril 2014, https://www.youtube.com/watch?v=fH-_oAocNbk, consultado en 26 de febrero de 2020) 


\section{.}

Con este testimonio podemos mirar el potencial emancipatorio del CAI y las posibilidades para obstaculizar el epistemicidio del que han sido objeto los pueblos indígenas no sólo en México, sino en todo el mundo.

El CAI, llevado a cabo en 2019, tuvo como entidades financiadoras a la Secretaría de las Culturas y Artes de Oaxaca que depende del gobierno estatal; de la Secretaría de Cultura y de la Dirección General de Vinculación Cultural que dependen del gobierno federal. Algunas entidades financiadoras extranjeras son la ONG Kultura Communication Desarrollo ubicada en Bilbao, Gobierno del País Vasco y la Agencia Vasca de Cooperación para el Desarrollo del Gobierno Vasco. Ojo de Agua Comunicación, una organización histórica ${ }^{15}$ que ha trabajado con el fortalecimiento de los procesos de comunicación en el estado de Oaxaca, también apoyó al CAI con becas para las y los participantes indígenas. Esto indica que los vínculos entre márgenes y centro son porosos y tienen puntos de encuentro que permiten las negociaciones, en este caso los apoyos financieros gubernamentales encarnan de manera clara estas negociaciones.

A continuación presento el cartel que publicaron los organizadores del CAI 2019 en su página de Facebook, donde enumeran los pasos para aplicar al Campamento y también donde aparecen los logos de las instituciones financiadoras que mencioné en el párrafo anterior.

Imagen 1. Pasos para aplicar al CAI Octava Edición 2019

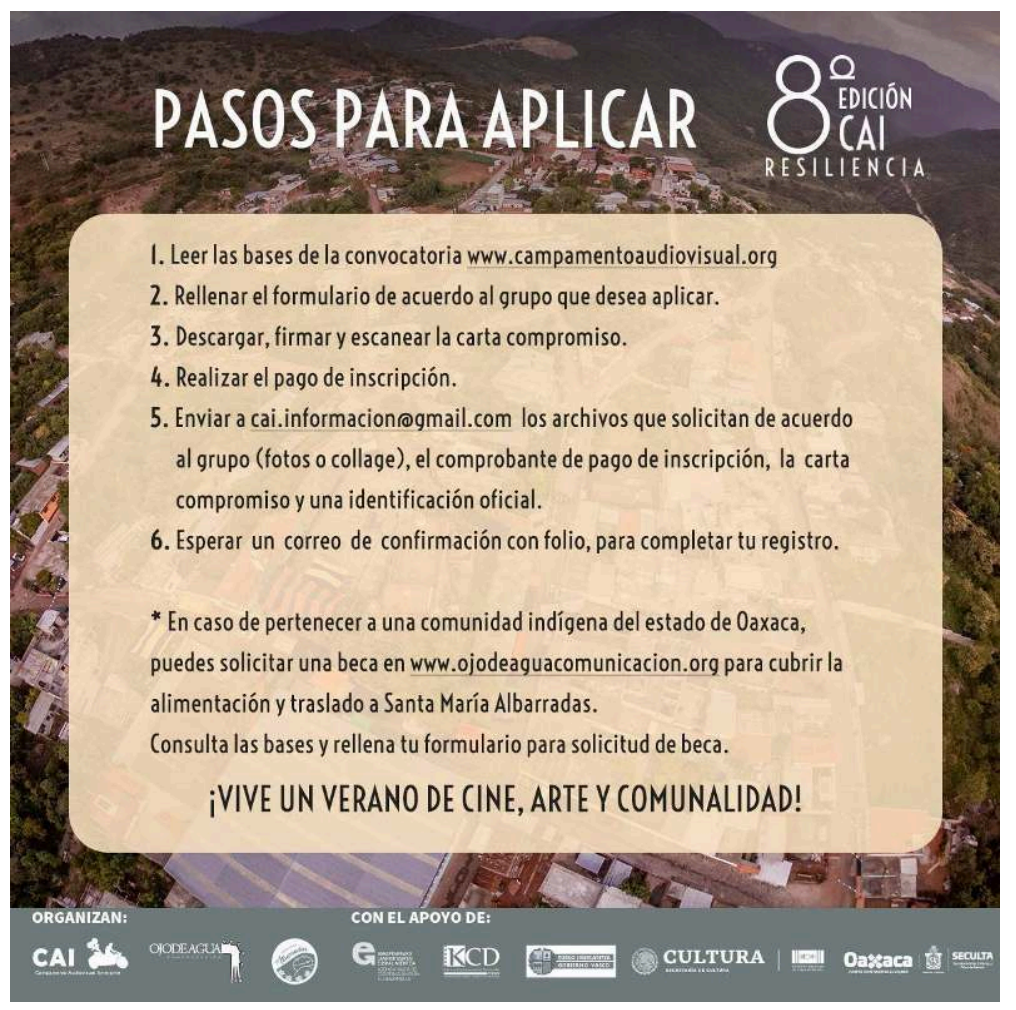

Cartel tomado de la página de Facebook del Campamento Audiovisual Itinerante

Podemos cerrar este apartado señalando que el CAI es un espacio de enseñanzaaprendizaje del quehacer audiovisual y cinematográfico desde las visiones locales, además reproduce no solo discursivamente la comunalidad, sino que al realizarse en comunidades de la sierra Norte de Oaxaca, logra que los participantes, aunque 
fugazmente, vivan las maneras de organizarse, de hacer política y la dimensión ritual en las comunidades.

\section{Conclusiones}

51 Es de importancia central mencionar una cuestión de contexto que permea históricamente las iniciativas comunitarias entre los ejemplos presentados en este texto. Las condiciones sociales y políticas de la región de la Sierra Norte de Oaxaca, permitieron que, aún bajo una situación de colonialismo interno donde ha prevalecido la discriminación por parte de la sociedad envolvente hacia los pueblos indígenas, pudiera emanar la comunalidad como un pensamiento indígena local que describe la vida de los pueblos. La noción de colonialismo interno para González Casanova P. (2006), está ligada en su origen a fenónemos de conquista donde el Estado absorbe a los pueblos y minorías, quienes: "se encuentran en situación de desigualdad frente a las elites de las etnias dominantes y de las clases que las integran" $(2006,410)$.

52 A pesar de las situaciones provocadas por el colonialismo interno en diferentes países, las luchas indígenas en México y en América Latina estaban siendo configuradas desde las décadas de 1970 y 1980 en que los conflictos sociales contemporáneos no sólo fueron políticos, sino que también tuvieron un fuerte peso en lo cultural, como lo explica Melucci A.: "el derecho de ser reconocido como diferente es una de las más profundas necesidades en la sociedad posindustrial o posmaterial" (1989:63, traducción libre).

53 A través de este artículo, mostré algunos de los ecos que la comunalidad, categoría creada desde los márgenes históricos, ha provocado en el estado de Oaxaca y cómo algunos sujetos, han reproducido sus elementos constitutivos en proyectos educativos y de gestión cultural.

Ejemplifiqué lo anteiror a través de dos casos: la UACO y el CAI. Proyectos surgidos en los márgenes que tienen momentos de cruces con el "centro". En el caso de la Universidad Autónoma Comunal de Oaxaca observamos cómo el proyecto pasó por la Legislatura del estado de Oaxaca y fue aprobado para ser una universidad "reconocida" por el Estado, aunque no siguiendo preceptos rígidos elaborados desde las entidades gubernamentales, sino con nuevas propuestas "desde abajo" que se espera que desemboquen en procesos educativos, donde la producción y sistematización de conocimientos pueda ser realizada por los pueblos. En el contexto de la pandemia derivada del COVID-19, la UACO no ha podido iniciar las primeras gestiones para comenzar sus trabajos, será importante dar continuidad al proceso que seguirá cuando la pandemia acabe ${ }^{16}$.

En el caso del Campamento Audiovisual Itinerante observamos cómo el proyecto también ha tenido cruces con el "centro" a través de los financiamientos que ha recibido de la Secretaría de las Culturas y Artes de Oaxaca que es parte del gobierno estatal y de la Secretaría de Cultura y de la Dirección General de Vinculación Cultural que son parte del gobierno federal. Y aunque en la entrevista con Damián López, él manifestó que para este año se pretende no tener los apoyos del gobierno federal ni estatal, años anteriores sí hubo inyección de recursos gubernamentales.

Es decir, en las dimensiones de lo legal y de los financiamientos, ambos casos tienen cruces o puntos de encuentro con el "centro", el cual es representado por diferentes entidades, prácticas, discursos, dispositivos, sujetos y está en constante transformación 
y recomposición (Das V. y Poole D., 2008). Lo que nos muestran estas experiencias es que el Estado, en algunas ocasiones y parcialmente, entabla negociaciones hasta cierto punto flexibles con los pueblos y comunidades indígenas. Es importante pensar cómo el "centro", encarnado en el Estado, para nuestro caso, apoya estas iniciativas que vienen desde los márgenes y cómo logra aceptar que la comunalidad sea un eje rector y filosófico de estos proyectos. Esto nos habla de un traslape o de una zona de contacto donde "centro" y "márgenes" logran llegar a acuerdos y negociaciones.

\section{BIBLIOGRAFÍA}

Entrevista con Damián López, Ciudad de Oaxaca, 2 de marzo de 2020. Realizada por la autora.

Entrevista con Arturo Guerrero, Ciudad de México, 19 de junio de 2020. Realizada por la autora.

Albert, Bruce, «Introdução. Cosmologias do contato no Norte-Amazônico», en Bruce Albert y Alcida Ramos (Orgs.), Pacificando o branco. Cosmologias do contato no Norte-Amazônico, São Paulo, Editora UNESP/Imprensa Oficial do Estado, 2000.

Aquino, Alejandra, «La comunalidad como epistemología del Sur: Aportes y retos», Cuadernos del Sur, 18 (34), 2013, p. 7-19.

Barabas, Alicia, «Un acercamiento a las identidades de los pueblos indios de Oaxaca», Amérique Latine Histoire et Mémoire. Les Cahiers ALHIM, 10, 2004 http://journals.openedition.org/alhim/105 Consultado el 24 junio 2020

Bartolomé, Miguel, Gente de costumbre y gente de razón. Las identidades éticas en México, México, Siglo XXI, 1997.

Bunzel, Ruth, Chichicastenengo: A Guatemalan Village, Seattle, University of Washington Press, 1952.

Comaroff Jean y Comaroff John, Theory form the South or, How Euro-America is evolving toward Africa, Boulder, Paradigm Publishers, 2012.

Constitución Política del Estado Libre y Soberano de Oaxaca (reformada mediante Decreto Num. 1489 aprobado por la LXIV Legislatura el 11 de marzo del 2020), https://www.congresooaxaca.gob.mx/ legislaciones/legislacion_estatal, página consultada el 23 de junio de 2020.

Das, Veena y Poole, Deborah, Anthropology in the Margins of the State, New Mexico, School of American Research Press, 2004.

Das, Veena y Poole, Deborah, «El estado y sus márgenes. Etnografías comparadas», Cuadernos de Antropología Social 27, 2008, p. 19-52.

Díaz, Floriberto, «Comunidad y comunalidad», en Sofía Robles y Rafael Cardoso (Eds.), Floriberto Díaz. Escrito. Comunalidad, energía viva del pensamiento mixe, México, UNAM, 2007.

González Casanova, Pablo, «Colonialismo interno (una redefinición)», en A. Boron, J. Amadeo y S. González (Comps.), La teoría marxista hoy, Buenos Aires, CLACSO, 2006.

Guerrero, Arturo, «La comunalidad como herramienta: una metáfora espiral», Cuadernos del Sur, 18 (34), 2013, p. 39-55. 
Flores, Rocío, «Proyectan Universidad Comunal en Oaxaca», Oaxaca Media, 9 de agosto de 2019, http://www.oaxaca.media/cultura/proyectan-universidad-comunal-en-oaxaca/, página consultada el 22 de febrero de 2020.

Flores, Rocío, «Aprueban creación constitucional de la Universidad Comunal de Oaxaca», Oaxaca Media, 16 de enero de 2020, http://www.oaxaca.media/cultura/aprueban-creacionconstitucional-de-la-universidad-comunal-de-oaxaca/, consultado el 22 de febrero de 2020.

Haraway, Donna, «Ciencia, cyborgs y mujeres. La reinvención de la naturaleza», Madrid, Cátedra, 1995.

Instituto Nacional de Estadística y Geografía (INEGI), Censo de 2010 y de 2015, https:// www.inegi.org.mx/datos/, página consultada el 22 de junio de 2020.

La Coperacha, «¿Qué es la Comunalidad?», La Coperacha, Ciudad de México, 16 Mayo 2016, https:// www.youtube.com/watch?v=zWmLEK29-NI, página consultada el 22 de febrero de 2020.

Lomnitz, Claudio, «Insoportable levedad», Fractal n 2, julio-septiembre, año 1, volumen I, 1996, pp. 51-76.

Maldonado, Benjamín, «Introducción», en: Jaime Martínez, Comunalidad y Desarrollo, México, CONACULTA/CAMPO, 2003.

Maldonado, Benjamín, «Comunidad, comunalidad y colonialismo en Oaxaca. La nueva educación comunitaria y su contexto», Oaxaca, CSEIIO/CEEESCI/CEDELIO/Secretaría de Asuntos Indígenas/ Universidad de Leiden, 2011.

Manzo, Diana, «Presentan la Universidad Autónoma Comunal de Oaxaca; tendrá 15 centros», La Jornada, 3 de septiembre de 2020, https://www.jornada.com.mx/2020/09/03/estados/025n1est, página consultada el 12 de septiembre de septiembre de 2020.

Martínez, Jaime, «Comunalidad y Desarrollo», México, CONACULTA, 2003.

Martínez, Jaime, «Eso que llaman comunalidad», México, CAMPO/Fundación Harp Helú/Secretaría de Cultura/Culturas Populares, 2010.

Matías, Pedro, «Con filosofía de comunalidad, arranca la Calenda Audiovisual», Página 3, 14 de julio de 2018, https://pagina3.mx/2018/07/con-filosofia-de-comunalidad-arranca-la-calendaaudiovisual/, página consultada el 22 de febrero de 2020.

Matías, Pedro, «Propone diputada crear Universidad Comunal de Oaxaca», Proceso, 22 de agosto de 2019, https://www.proceso.com.mx/596897/propone-diputada-crear-universidad-comunalde-oaxaca, página consultada el 22 de febrero de 2020.

Matías, Pedro, «Aprueban la creación de la Universidad Autónoma Comunal de Oaxaca», Proceso, 16 de enero de 2020, https://www.proceso.com.mx/614535/aprueban-la-creacion-de-launiversidad-autonoma-comunal-de-oaxaca, página consultada el 23 de febrero de 2020.

Melucci, Alberto, «Um objetivo para os movimentos sociais?», Lua Nova Revista de Cultura e Politica 17,1989 , p. 49-66.

Mesri Hashemi-Dilmaghani, Parastoo Anita, «Una propuesta para la promulgación de una ley de justicia indígena en el estado de Oaxaca», Nueva antropol [online], vol. 26, $\mathrm{n}^{\circ} 78$ [citado 2020-06-24], 2013, pp.119-140.

Nash, Manning, «Political Relations in Guatemala», Social and Economic Studies 7, 1958, 65-75. Nava, Elena, «Comunalidad: semilla teórica en crecimiento», Cuadernos del Sur, 18 (34), 2013, p. 57-69. 
Nava, Elena, «La Comunalidad oaxaqueña: lucha y pensamiento indígena», en Pedro Canales y Sebastião Vargas (Eds.), Pensamiento Indígena en Nuestramérica. Debates y Propuestas en la Mesa de Hoy, Santiago de Chile, Ariadna Ediciones, 2018.

Tax, Sol, «The Municipios of the Midwestern Highlands of Guatemala», 3-4 American Anthropologist 39, 1937, p. 423-444.

Voces de Oaxaca, «Luna Marán: El CAI 2014 ya abrió su convocatoria», Voces de Oaxaca Periodismo Colectivo, 3 de abril de 2014, https://www.youtube.com/watch?v=fH-_oAocNbk, página consultada el 26 de febrero de 2020.

Wagley, Charles, The Social and Religious Life of a Guatemalan Village, Wisconsin, American Anthropological Association Memoir 71, 1949.

Williams, Raymond, Marxismo y literatura, Barcelona, Ediciones Península, 1988.

Wolf, Eric, Sons of the Shaking Earth, Chicago, Chicago University Press, 1959.

\section{NOTAS}

1. Con nuevas voces me refiero a nuevas configuraciones de jóvenes y adultos (no necesariamente indígenas de la Sierra Norte de Oaxaca pero sí mayoritariamente originarios de ese lugar), que tienen en común el interés de resignificar y reproducir de diferentes formas la comunalidad y sus prácticas. Estas nuevas voces están construyendo espacios socio-políticos que les permiten la sistematización de conocimientos locales y la generación de una legitimidad de estos.

2. Con trabajo de campo no continuo de larga duración, me refiero a un trabajo de campo que no es continuo debido a los compromisos de docencia adquiridos en la universidad y es de larga duración, pues desde el año 2001 en que llegué por primera vez al estado de Oaxaca a realizar mi trabajo de campo para mi tesis de licenciatura, casi cada año viajo al estado de Oaxaca de una a cinco veces.

3. La Sierra Norte de Oaxaca es una de las ocho regiones en las que se divide el estado de Oaxaca.

4. Ver: Martínez Luna F., 2013; Maldonado Alvarado B., 2002, 2011; Rendón J., 2003; Manzo C., 2011; entre otros.

5. En este texto haré mención de la categoría de hegemonía, la cual retomo de Gramsci, quien inclinado por la "superestructura" en Marx construyó este término. Para Williams R. (1988), la hegemonía en Gramsci se expresa en formas políticas, sociales y culturales coercitivas.

6. Cuadernos del Sur. Revista de Ciencias Sociales, Año 18, \#34, Enero-Junio 2013. Oaxaca, México.

7. En un texto de 1997, Miguel Bartolomé, hablando desde Oaxaca, explica que es importante profundizar en el concepto de "líderes" pues para las sociedades mesoamericanas habría que entender hasta dónde la representación a través de "líderes" es legítima (p.168). En las mismas comunidades la categoría de "líder" es criticada por centrarse en el individuo. Bartolomé lo explica de la siguiente manera: "No existe una delegación de la autoridad colectiva en los cargueros; estos no son líderes, representantes o conductores de sus paisanos, sino guías o reguladores de la interacción social, política y económica. Es decir gente encargada de que la vida colectiva se mantenga dentro de los cauces que ha seguido siempre, pero que no tienen la capacidad de cambiarla." (p.169).

8. Tanto la CODREMI como la ODERNASIJ son organizaciones que se crearon a la par que la Unión de Pueblos del Rincón y de la Asamblea de Autoridades Zapotecas, ambas organizaciones de la Sierra Norte, con objetivos muy parecidos. 
9. Se le llama tequio al trabajo colectivo no asalariado que es realizado en las comunidades indígenas en Oaxaca (por ejemplo, la construcción de una clínica o un camino, la limpieza de las brechas que marcan los límites entre municipios, etc.). Conforme avanzan los procesos de inserción de los pueblos en la economía capitalista (asalariada), el tequio pierde fuerza en algunos espacios. Sin embargo, sigue siendo uno de los ejes principales de los sistemas organizativos de las comunidades.

10. Un ejemplo ilustrativo puede ser la idea de comunalizar que nos muestran algunos medios de comunicación comunitarios donde "los actores están haciendo del medio un espacio común donde los pueblos son capaces de practicar la comunalidad en sus diversas escalas, ya sea hacia adentro de las mismas estructuras organizativas de los medios o en relación con sus vínculos con la comunidad." (Nava E., 2018: 146)

11. Sobre el sistema de cargos existe una amplia bibliografía que data de la década de 1930 con los primeros estudiosos del tema como Tax S. (1937), Wagley C. (1949) o Bunzel R. (1952). Posteriormente, más autores como Wolf E. (1959) o Nash J. (1958) también abordan el tema. Es central dejar claro que el sistema de cargos no solo es un sistema político, sino que abarca lo religoso también.

12. Para más información sobre la visita de Andrés Manuel López Obrador a Guelatao ver: https://www.jornada.com.mx/ultimas/politica/2019/03/21/amlo-conmemora-hoy-en-

guelatao-213-aniversario-de-benito-juarez-4681.html

13. El 21 de marzo de cada año se celebra en México el natalicio de Benito Juárez, quien fue presidente de México varias veces durante el siglo XIX. Se le conoce como el "Benemérito de las Américas”, nació en Guelatao de Juárez en 1806. Por este motivo, el actual presidente viajó a Guelatao, para celebrar el natalicio de Benito Juárez en su lugar de origen.

14. La Academia de la Comunalidad, a la cual yo también pertenezco, tuvo como propósitos iniciales: “a) Reunir a todos aquellos que estudian y difunden valores profundos de la comunalidad, o en sus programas de labor ven la necesidad de profundizar el conocimiento de la práctica y filosofía del concepto. b) Articular esfuerzos aislados que giran en torno a la práctica comunalitaria, abriendo espacios que permitan el enriquecimiento del concepto a través de la reflexión y el intercambio, como de su puesta en práctica en áreas no comunalizadas." (Anteproyecto de la Academia de la Comunalidad, abril de 2012)

15. Ojo de Agua Comunicación es una organización que ha trabajado desde inicios de la década de 1990 con el fortalecimiento de los procesos de comunicación indígena en el estado de Oaxaca. En el contexto del Programa de Transferencia de Medios Audiovisuales a Comunidades y Organizaciones Indígenas, llevado a cabo en 1989 por el Instituto Nacional Indigenista (INI), es que se fundó el Centro de Video Indígena en la ciudad de Oaxaca en 1994, el cual se transformó, en 1998, en Ojo de Agua Comunicación S.C.

16. El 2 de septiembre de 2020 se presentó públicamente la Universidad Autónoma Comunal de Oaxaca en una ceremonia llevada a acabo en Santa María Tlahuitoltepec Mixe, donde Jaime Martínez Luna fue designado como rector de la UACO (Manzo D., La Jornada, 2020)

\section{RESÚMENES}

En este artículo se analiza cómo la comunalidad, como una categoría creada en la década de 1980 por dos intelectuales indígenas en el estado de Oaxaca (México), no se queda estática en el tiempo, sino que atraviesa generaciones emergiendo como eje rector de nuevos proyectos 
educativos y culturales. A través de dos ejemplos, la Universidad Autónoma Comunal de Oaxaca y el Campamento Audiovisual Itinerante, se muestra cómo la comunalidad ha sido retomada por nuevas voces, fomentado la sistematización propia y local del conocimiento y estableciendo relaciones diversas con entidades vinculadas al Estado.

Este artigo analisa como a comunalidad, uma categoria criada na década de 1980 por dois intelectuais indígenas no estado de Oaxaca (México), não permanece estática no tempo, mas atravessa gerações aparecendo como eixo reitor de novos projetos educativos e culturais. Por meio de dois exemplos, a Universidade Autônoma Comunal de Oaxaca e o Acampamento Audiovisual Itinerante, se mostra como a comunalidad foi absorbida por novas vozes, tem promovido a sistematização própria de conhecimento local e o estabelecimento de relações diversas com entidades vinculadas ao Estado.

This article discusses how comunalidad, a category created in the 1980 s by two indigenous intellectuals in the state of Oaxaca (Mexico), does not remain static over time, but passes through generations and emerges as a guiding axis of new educational and cultural projects. By way of two examples, the Autonomous Communal University of Oaxaca and the Itinerant Audiovisual Camp, the article shows how comunalidad has been taken up by new voices, has promoted grassroot and local systematization of knowledge and helped to establish diverse relationships with State institutions.

\section{ÍNDICE}

Keywords: comunalidad, Oaxaca, Indigenous Peoples, indigenous intellectuals Palavras-chave: comunalidad, Oaxaca, Povos Indígenas, intelectuais indígenas

Palabras claves: comunalidad, Oaxaca, Pueblos Indígenas, intelectuales indígenas

\section{AUTOR}

\section{ELENA NAVA MORALES}

Investigadora del Instituto de Investigaciones Sociales de la UNAM. Doctora en Antropología Social por la Universidad de Brasilia, Brasil. Pertenece al Sistema Nacional de Investigadores Nivel 1. Su último libro es Totopo al aire: Radio comunitaria y comunalidad en el istmo de Tehuantepec (CIESAS). Temas de interés: medios de comunicación comunitarios-indígenas, redes sociodigitales y pueblos indígenas, procesos de resistencia indígena y metodologías de la antropología. 